\title{
AC 2008-2605: WHY HAVE ENGINEERING FIELDS BEEN SLOWER TO CHANGE THAN OTHERS?
}

Caroline Hayes, University of Minnesota 


\title{
Why are Engineering Fields Slower to Change than Others?
}

"Pioneers are the people lying face down with the arrows in their backs." -- Anonymous.

\begin{abstract}
Women continue to be underrepresented at all levels in engineering fields, even relative to other science, technology or math fields. This paper explores what, if anything is different about engineering fields that may be holding them back. It does so by examining and combining data from national data sets on gender distributions of students and faculty in a variety of science and engineering fields. The paper focuses on engineering at colleges and universities because of the role which these institutions have in inspiring both women and men to choose engineering as a field, and their potential to change the composition and size of the future workforce. Findings include that for fields dominated by men in the 1960s 1) those fields with the highest (or lowest) proportions of women students in the 60's still have the highest (or lowest) proportions of women students today, and 2) the proportion of women students is highly correlated with the proportion of women faculty in a field. This may suggest that increasing the number of women faculty may be a strategy for more rapidly attracting women students into a field. Finally, the paper raises additional questions we need to be asking as a community to uncover barriers and to develop intervention strategies that can attract more talented engineers of both genders, and improve the climate for all.
\end{abstract}

\section{Introduction}

Over the last 50 years, there have been great increases in the number of women in some traditionally male dominated professions such as law and medicine. However, women have not entered all such professions at the same rates. For example, medical schools in the United States graduated 45 percent $^{1}$ women in 2004, while computer science schools graduated only 25 percent women Bachelors of Science and 21 percent women PhDs. ${ }^{2,13}$ Literature on women in STEM (science, technology, engineering and math) fields documents many factors that have made it difficult for women to gain acceptance in male dominated professions including a dearth of female role models, ${ }^{3,4}$ implicit gender biases, ${ }^{3,4,5,6}$ and reluctance (often unconscious) from people in positions of power to include women in their informal networks ${ }^{7}$ or act as their professional allies. ${ }^{8}$

However, since these factors apply to all historically male dominated disciplines, they do not adequately explain why fields such as law and medicine have changed more rapidly than engineering. Is there something inherently different about the nature of engineering work in engineering organizations that has made engineering less appealing to women? It is essential that we understand and overcome the barriers for women in engineering because we need to develop as much potential engineering talent as possible if we are to have a sufficient number of engineers available to continue supporting a high-tech economy ${ }^{11}$. This is not simply a matter of fairness; the quality of the engineering profession will fail to reach its full potential if it continues to make full use of only a fraction of the talent pool. White males comprise less than half of the US population. In order to be fully productive and competitive engineering fields must draw from a much larger cross section of potential talent. 


\section{The Current Status of Women across Professions}

Even as far back as the beginning of the $20^{\text {th }}$ century, women have been earning degrees at colleges and universities, although the percentages of women doing so were relatively small between 1900 and $1960 .{ }^{4}$ However, the change between 1960 and today has been dramatic with particularly rapid changes occurring in the 1970's. In 1960, slightly more than 40 percent of the bachelor's degrees and roughly $8 \%$ of the PhDs were awarded to women at American educational institutions. Now, more than 60 percent of all bachelors and almost 50 percent of the PhDs are awarded to women in the US. ${ }^{9,13}$

However, Mason and Ekman ${ }^{4}$ report that even in fields such as law, medicine and publishing in which women are relatively well represented among the graduates, women are not as well represented in the higher prestige, higher paying segments of these fields and they are very rare in leadership roles such as upper management. Thus, to get a whole picture of the status of women in any field it is necessary to look at more than just numbers of graduates. One must also examine what happens to them after graduation. What type of positions do they hold? What salaries do they command? Are there opportunities for them to take leadership roles capable of shaping a field? ${ }^{9} \quad$ Unfortunately, in most fields there are relatively few women in leadership positions. ${ }^{3}$ It is partly a "pipeline" issue in which there are not enough women graduates old enough to be in these positions yet, and partly that the pipeline is very leaky. ${ }^{10}$ Women are not necessarily hired, retained or promoted at the same rates as men. ${ }^{9}$ Nelson reports a study stating that women in science and engineering are tenured at half the rate as men. ${ }^{16}$ Additionally, some women drop out voluntarily, citing a variety of reasons from a chilly climate, to overwhelming pressure from a combination of professional and family demands. ${ }^{9}$ Those that do stay and are promoted are not, on average, paid as well as their male counterparts; even after controlling for variables including institution type, discipline, rank, teaching and publications, the unexplained salary differences between male and female faculty are still about 9 percent. ${ }^{12}$ Addressing these issues starts with an understanding of what is happening at multiple levels within a field. This paper will provide a multi-level examination (bachelor's, $\mathrm{PhD}$ and multiple faculty ranks) of the status of women in engineering at colleges and universities and how it has changed over time.

\section{Past and Current Status of Women in Engineering Relative to Other Fields}

Bachelor's degrees in engineering. Figure 1 shows the National Science Foundation data ${ }^{13}$ on the percentages of women graduating between 1966 and 2004 with bachelor's degrees in a variety of fields, including engineering. Some fields such as psychology, social sciences (economics, political sciences, sociology) and non-S\&E fields became female dominated starting in the 1970s and 1980s.

"Non-S\&E fields" is the National Science Foundation's designator for non-science and engineering fields; these include most educational training programs. The black line marked with black squares in Figure 1 is the average number of bachelor's degrees earned by women across all fields offered in the US, most of which are not shown on the graph. Note that this baseline has changed over time. Women earned 43.6 percent of all bachelor's degrees in 1966, but by 2004 they were earning 57.6 percent of them. Many fields have greatly increased their percentages of women bachelor's graduates, most notably Psychology; the biological and 
agricultural sciences; earth, atmosphere and ocean sciences; and the physical sciences which include Astronomy, Chemistry and Physics.

Engineering is represented by the line at the very bottom of Figure 1. In 1966 women receiving engineering bachelor's degrees were almost non-existent; they earned just 0.4 percent of all engineering bachelor's degrees. In absolute numbers, there were 146 women recipients out of a total of 35,862 awarded that year. By 2004 women were earning 20.5 percent of all engineering bachelor's degrees $(13,257$ out of a total 64,675$)$. However, this percentage is still well below other traditionally male dominated fields such as math and computer science, even given the recent losses in women applicants in computer science, and far below the baseline.

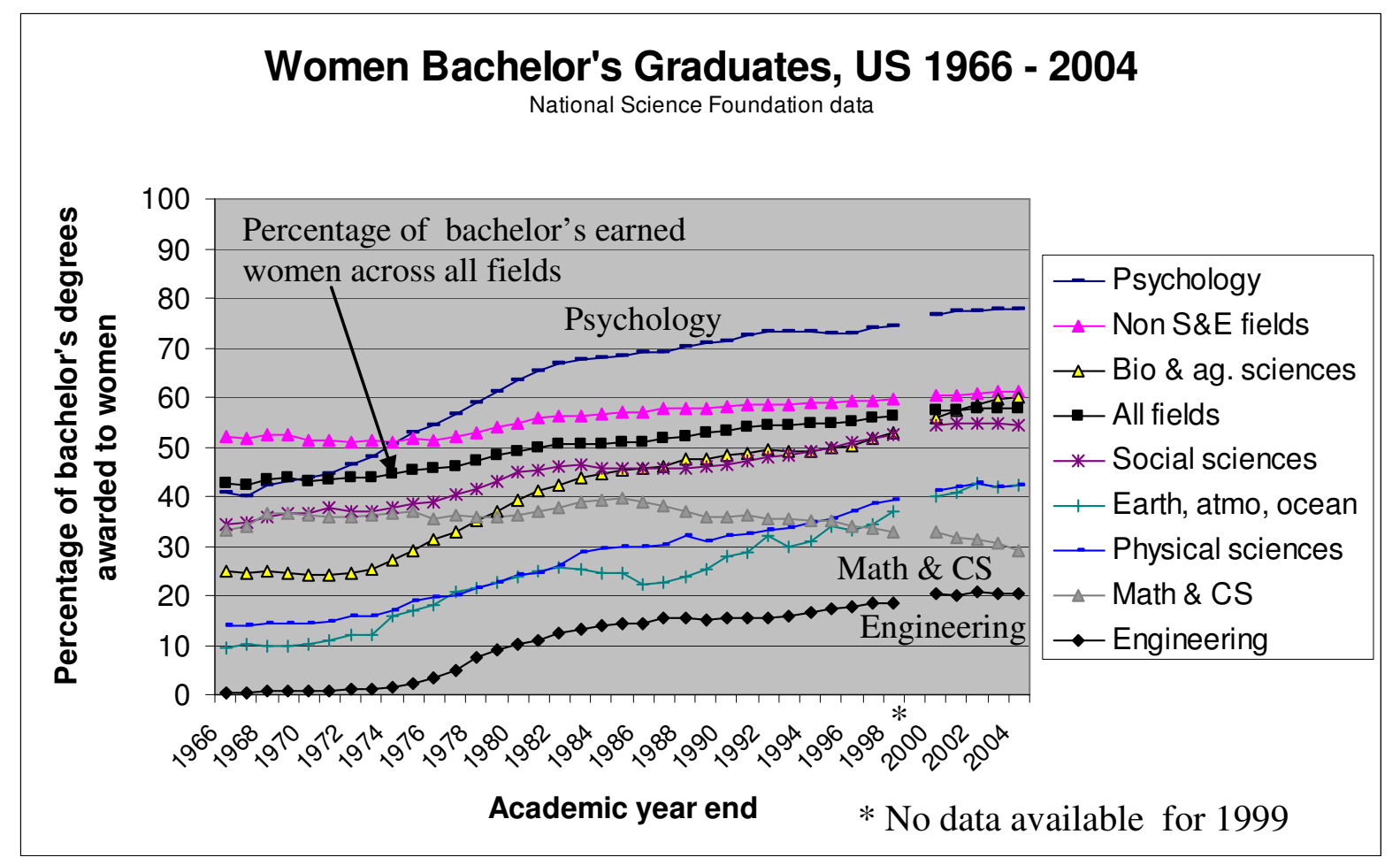

Figure 1: The status of women BS graduates in engineering relative to other fields, NSF data. ${ }^{13}$

On the positive side, all fields shown in Figure 1 have experienced significant increases in the percentage of women graduates, with particularly rapid advances occurring over a 10 year period between roughly 1973 and 1983. These rapid changes were possibly fueled by the women's rights moment which had much momentum during that same time period. This period of rapid growth was followed in many fields by a period of slower growth or, in the case of Math and Computer Science, a significant drop in the number of women bachelor's degrees. It is not clear why this is so, but these data may imply that rapid change is possible, but that a sustained focus on gender equality may be necessary to sustain the rate of change or prevent backsliding.

Finally, it is worth commenting on the very large number of women bachelor's graduates in Psychology (77.8 percent in 2004). While this is not necessarily a bad thing, it is not necessarily 
a good thing either if diversity is important for the health of a field and the satisfaction of the people in that field. ${ }^{14}$

PhDs in engineering. Figure 2 shows the National Science Foundation data ${ }^{13}$ on the number of women $\mathrm{PhD}$ recipients for the same set of fields depicted in Figure 1. Again, engineering is at the very bottom of the graph, with 0.3 percent of engineering PhDs ( 8 out of 2,301) awarded to women in 1966 and 17.6 percent in 2004 (1,014 out of 5,776). As a baseline comparison, the total percentage of women receiving PhDs across all fields was 11.6 percent in 1966, and it has grown to 45.3 percent in 2004.

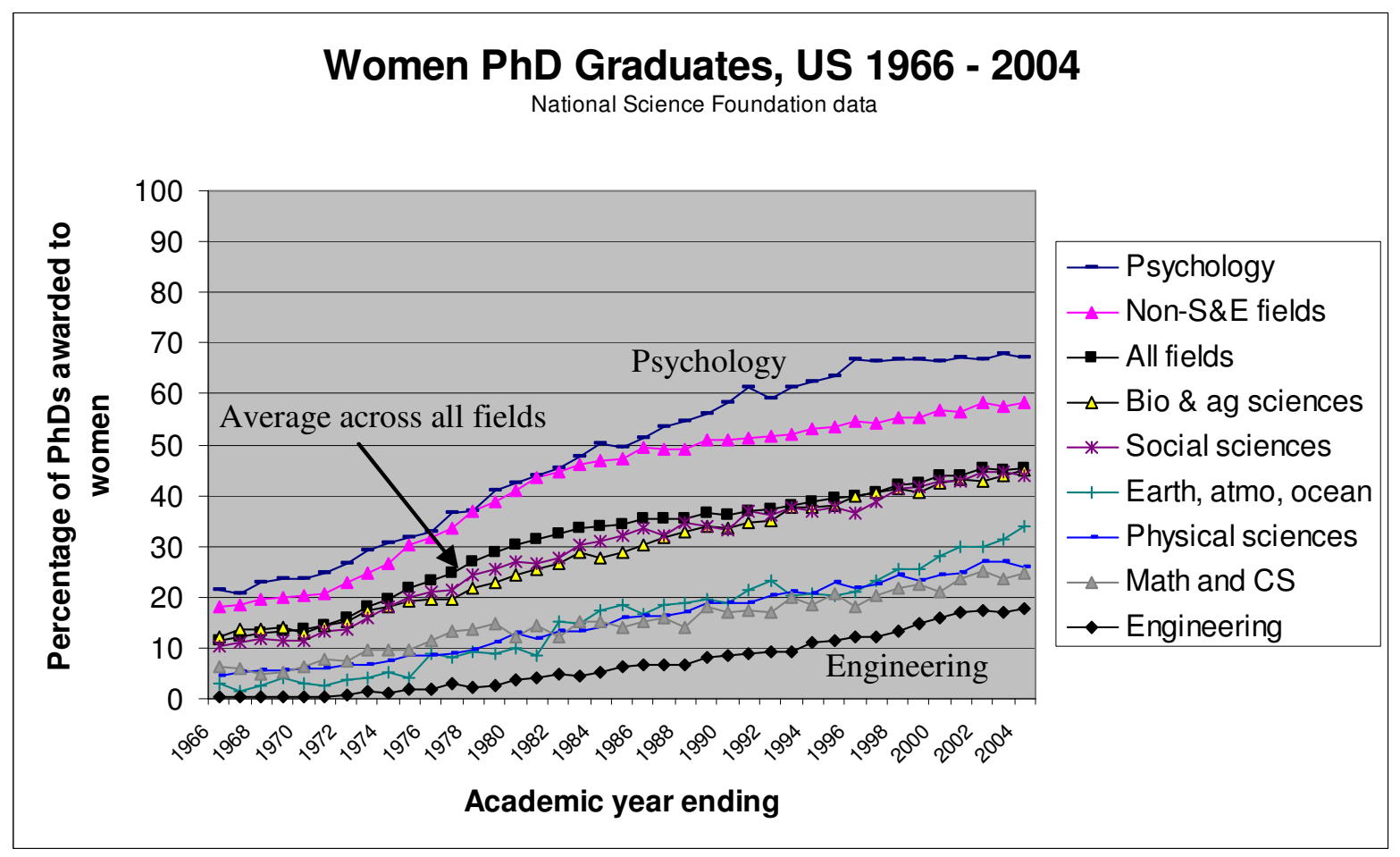

Figure 2: The status of women PhDs in engineering relative to other fields, NSF data. ${ }^{13}$

Encouragingly, the percentages of $\mathrm{PhDs}$ awarded to women have grown in all of the fields represented in Figure 2, including Computer Science where the percentage of women bachelors (and degrees overall) has been falling since the mid 1980s. Psychology awarded 67 percent of its $\mathrm{PhDs}$ to women in 2004. The social sciences, biological and agricultural sciences have reached almost equal proportions of men and women PhDs graduates. However, earth, atmosphere, and ocean sciences; the physical sciences; math and computer science; and engineering fall far below that level with engineering trailing the pack. This is important because the leaders in science and engineering fields in both industry and academia often hold $\mathrm{PhDs}$. If there are few women $\mathrm{PhDs}$ in these areas now, there may be few women leaders in the near and medium-term future.

Interestingly, the rank order of all the fields shown in Figure 2 (with respect to percentages of women) has changed little since 1966. In other words, the fields that had the most women PhDs in 1966 still have the most women PhDs today, while fields like engineering that had the least 
women PhDs still have that least forty years later. The same is true to a lesser extent for bachelor's degrees (in Figure 1) except for Psychology (which has surpassed Non-S\&E fields) and Computer Science (which has dropped below many fields). Both Figure 1 and Figure 2 suggest that the relative percentage of women graduated in a field in the past predicts the relative percentages of women that will graduate from that field in the future, even a long time in the future. Does this imply that Engineering is destined to stay at the bottom for a long time to come? Or does it mean that it requires women to already be in a field in order to attract more women in the future? By focusing on education, hiring, retention and promotion of women at all levels, can engineering catch-up and surpass some of these other fields? Perhaps it means both; if we do not do the latter then we are doomed to the former.

Faculty in Engineering. While both male and female faculty can be role models and inspirations to all students the presence of women faculty in a field may be particularly important to prospective women students. The presence of women may signal to female students that they belong there, and their absence may signal that they do not. It can also send the message that a future career trajectory is possible since women can hold respected positions in that field. This is an important issue to study. There is, unfortunately, little publicly accessible long-term national data available on the percentages of women faculty in various professions. However, Nelson has recently published two national studies presenting data from $2002^{16}$ and $2007^{11}$ reporting the percentages of women and minority faculty in a wide range of science and fields.

Table 1 and Figure 3 show a combination of Nelson's 2002 data $^{13}$ on the national percentages of women faculty in various science and engineering fields, and the National Science Foundations' 2002 data $^{13}$ on $\mathrm{PhD}$ graduates for those same fields.

Table 1: Percentages of Women PhDs and Faculty at all ranks in selected fields.

\begin{tabular}{|l|r|l|r|r|r|r|}
\hline & $\begin{array}{l}\text { Total } \\
\text { PhDs } \\
2002 \\
\text { Discipline }\end{array}$ & $\begin{array}{l}\text { Women } \\
\text { PhDs } \\
\text { (NS) } \\
\text { (NSF) }\end{array}$ & $\begin{array}{l}\text { Assistant } \\
\text { Professor } \\
\text { (Nelson) }\end{array}$ & $\begin{array}{l}\text { Associate } \\
\text { Professor } \\
\text { (Nelson) }\end{array}$ & $\begin{array}{l}\text { lFull" } \\
\text { Professor } \\
\text { (Nelson) }\end{array}$ & $\begin{array}{l}\text { All } \\
\text { Faculty } \\
\text { Ranks } \\
\text { (Nelson) }\end{array}$ \\
\hline Chemistry (2003) & 2041 & $31.8 \%$ & $21.5 \%$ & $20.5 \%$ & $7.6 \%$ & $12.1 \%$ \\
\hline Math & 918 & $28.9 \%$ & $19.6 \%$ & $13.2 \%$ & $4.6 \%$ & $8.3 \%$ \\
\hline Computer Science & 807 & $20.6 \%$ & $10.8 \%$ & $14.2 \%$ & $8.3 \%$ & $10.6 \%$ \\
\hline Astronomy (2003) & 168 & $24.4 \%$ & $20.2 \%$ & $15.7 \%$ & $9.8 \%$ & $12.4 \%$ \\
\hline Physics & 1127 & $15.7 \%$ & $11.2 \%$ & $9.4 \%$ & $5.2 \%$ & $6.6 \%$ \\
\hline Chemical Engineering & 705 & $25.0 \%$ & $21.4 \%$ & $19.2 \%$ & $4.4 \%$ & $10.5 \%$ \\
\hline Civil Engineering & 626 & $19.2 \%$ & $22.3 \%$ & $11.5 \%$ & $3.5 \%$ & $9.8 \%$ \\
\hline Electrical Engineering & 1395 & $11.7 \%$ & $10.9 \%$ & $9.8 \%$ & $3.8 \%$ & $6.5 \%$ \\
\hline Mechanical Engineering & 827 & $11.6 \%$ & $15.7 \%$ & $8.9 \%$ & $3.2 \%$ & $6.7 \%$ \\
\hline Economics & 1024 & $27.4 \%$ & $19.0 \%$ & $16.3 \%$ & $7.2 \%$ & $11.5 \%$ \\
\hline Political Science & 938 & $42.0 \%$ & $36.5 \%$ & $28.6 \%$ & $13.9 \%$ & $23.5 \%$ \\
\hline Sociology & 565 & $61.1 \%$ & $52.3 \%$ & $42.7 \%$ & $24.3 \%$ & $35.8 \%$ \\
\hline Psychology & 3199 & $66.7 \%$ & $45.4 \%$ & $40.1 \%$ & $26.7 \%$ & $33.5 \%$ \\
\hline Biological Sciences & 5690 & $44.7 \%$ & $30.4 \%$ & $24.7 \%$ & $14.7 \%$ & $20.1 \%$ \\
\hline
\end{tabular}


The work reported in this paper will use these data to explore the question, "Is there a relationship between the percentage of women faculty in a field, and the percentage of women $\mathrm{PhD}$ students attracted to it?" Ideally, one should compare the percentage of women faculty in a field in a given year to the percentage of women $\mathrm{PhD}$ graduates four to six years later. One cannot make that comparison for Nelson's 2007 faculty data set because any $\mathrm{PhD}$ candidates attracted to a field in 2007 have not yet graduated. Nor can one do that for Nelson's 2002 data because NSF has not yet published the figures for PhD graduates for 2006/07. However, we hope to get an approximate idea of whether a relationship exists by examining the 2002 faculty data and the 2002 student data. It would also be useful to examine the proportions of $\mathrm{PhD}$ applicants, but to the author's knowledge, no such publicly accessible data set exists.

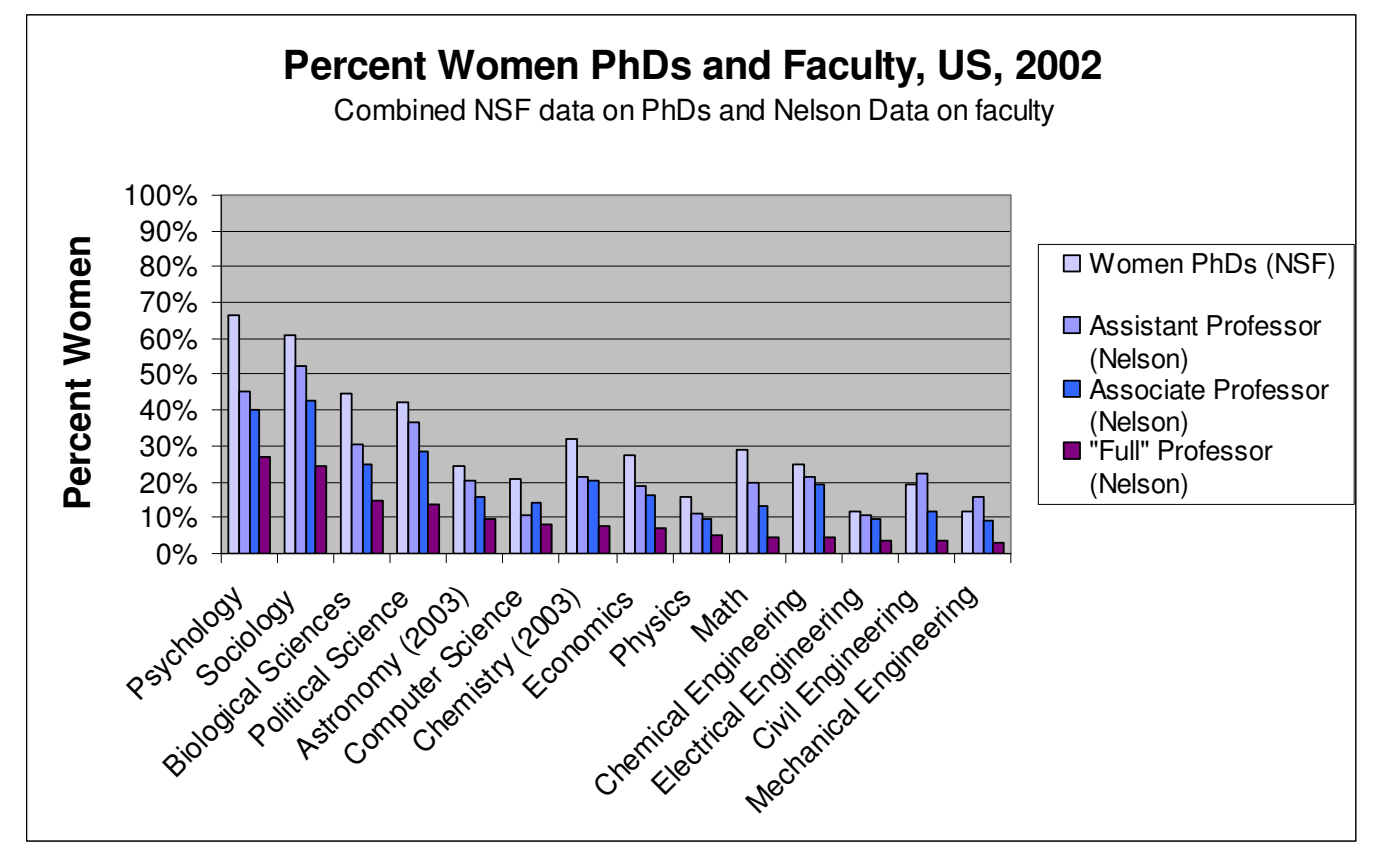

Figure 3: Women PhDs and faculty in various science and engineering fields.

Figure 3 provides a graphical representation of the data in Table 1. The disciplines in Figure 3 are sorted from largest to smallest percentages of women "full" professors. There are four engineering fields represented in Figure 2, Chemical, Civil, Electrical and Mechanical Engineering. All are clustered on the right (the bottom of the heap). Nationally, relatively few women choose to earn $\mathrm{PhDs}$ in engineering, and still fewer women who become professors with less at each rank. While it is not necessarily a bad thing if women choose to go into other professions, it is a loss in that professors are often role models who may influence career choices, and women faculty are not proportionally participating in that process. There are the fewest women at the rank of "full" professor; there are between 4 and 7 percent women engineering "full" professors nationally. ${ }^{11}$ As a baseline comparison, women made up 24 percent of all full professors in the US in $2003^{9}$ across all fields, and 39 percent of full time faculty across all ranks and fields. ${ }^{9}$

Marschke and colleagues present an analysis suggesting that gender parity may never occur in for full-time faculty in some universities without significant changes. ${ }^{17}$ Their analysis for a 
specific university predicted a steady state of 34 percent women faculty requiring 57 years to reach that state, given current hiring and retention practices. The 2007 AAUP report on faculty gender equity indicators states that existing and persistent lack of gender equity in higher education "place(s) serious limitations on the success of educational institutions."

Returning to Figure 3, it is interesting to note that the two fields with the smallest percentages of women "full" professors: Civil and Mechanical, have higher percentages of women at the assistant level than one would expect given the percentages of recent $\mathrm{PhDs}$. This may be the result of aggressive recruiting and hiring of women. In Nelson's 2007 data, this pattern appears for all four of the engineering fields represented, which may indicate that more engineering fields are now aggressively hiring women at the lower ranks.

Table 2: Correlations between percentages of women PhDs and women faculty (Table 1 data).

\begin{tabular}{|l|r|}
\hline & Women PhDs \\
\hline Women PhDs & 1.00 \\
\hline Assistant Prof & 0.94 \\
\hline Associate Prof & 0.97 \\
\hline "Full" Prof & 0.95 \\
\hline All Faculty Ranks & 0.96 \\
\hline
\end{tabular}

Table 2 shows strong correlations (0.94 to 0.97) between the number of women PhD graduates, and the number of faculty in that field at any rank. Figure 4 depicts this relationship in a scatter plot of the data in Table 1. This shows that although the percentage of women PhDs graduates is typically larger than the percentage of women faculty, they are proportional.

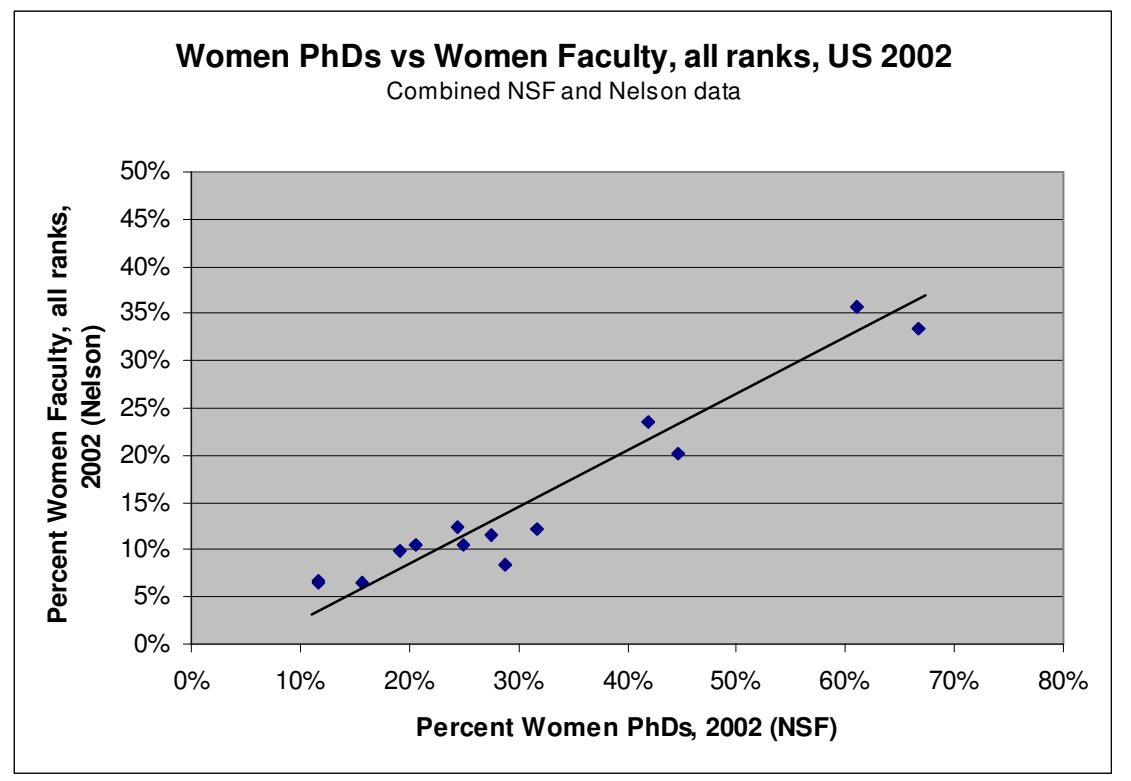

Figure 4: Women PhDs vs. women faculty. Scatter plot of data from Table 1. 
Does the proportion of faculty impact the proportion of students or the reverse? Probably some of both occur. While more investigation is needed to develop an explanation, the presence and number of women faculty and leaders in a field may have a significant conscious or unconscious influence on students. If more national data continues to be collected on gender rations of faculty over time, it may be possible to explore this relationship more deeply. The Nelson data also show that there are some very large differences between institutions in the proportion of women faculty in any given field. From this one might infer that the climate, culture and attitude at individual institutions may be very different from each other. Local factors may be very important in creating an equitable (or inequitable) climate. It may also imply that change can happen at individual institutions without requiring attitudes in the whole field to change first.

\section{In What Ways Might Engineering Fields Differ from Others?}

One obvious way in which engineering is different from other fields, which may initially sound trite, but may in fact not be, is that it has fewer women. It has fewer women students, fewer women faculty, and probably fewer women leaders (department head, dean, etc.). If indeed one needs women to get more women, as Figures 2 and 4 suggest, then it may require extraordinary efforts to change to rate at which women enter a field which currently has very few women. Yet, how did engineering get that way in the first place? Why did so few women decided to pursue engineering degrees and careers prior to 1966, and why are they still so underrepresented? Blum and Frieze ${ }^{14}$ present some very interesting studies of women in the Computer Science Department at Carnegie Mellon University which may provide important insights for engineering.

Margolis and Fisher ${ }^{15}$ performed an earlier study in this same department in the mid 1990s when it had a very gender imbalanced environment. They interview approximately 100 students found many differences between the male and female students in background, prior computer experience and computer programming skills, and feelings of satisfaction and inclusion in their current program. In the late 1990's the department made some changes to their admission standards. They kept standards for GREs and grades high, but they added an emphasis on leadership qualities and dropped requirements for prior programming experience. Additionally, they added a few "catch-up" courses to the curriculum to level-out background differences. By doing so, the department increased the percentage of women students from something less than 10 to over 30 percent, and greatly changed the culture. Blum and Frieze interviewed students during these changes and found as the environment became more gender balanced, many of the apparent differences observed by Margolis and Fisher between the male and female students began to fade. The background of both male and female students had become more diverse, and the level of satisfaction of both had increased.

These lessons may hold for engineering as well. If measures can be taken to improve the gender balance and culture of engineering, it may not only change its appeal for women but for men too. Those measures may include a range of approaches from taking proactive steps to increase the number of women in faculty (at all ranks) and leadership positions in engineering, to changing admission criteria and curriculum. There are likely other factors that should be considered, as will be discussed in the next section. 
Additional issues for future investigations which may influence and perpetuate the perception of engineering as an unappealing field for women include:

1. The perception of glamour associated with a field, and perpetuated in the media. Movies and television depict many hero doctors in the emergency room saving lives and hero lawyers in the courtroom defending justice for the underdog, but where are the hero engineers? If they are depicted at all, it is as nerdy variants of mad scientists who accidentally or intentionally invent devices that can be used for mass harm. Media can also perpetuate or break-down gender stereotypes associated with a profession.

2. Knowledge half-life: the rate of decay of knowledge relevance. This may impact the ease with which people taking time out for children can re-enter a field later. Medical knowledge is still considered to be mostly valid after a few years, but in more purely high-tech fields, such as engineering, what one knows now about current technology may not be very relevant 3 to 5 years later.

3. Amount of research funding, and salaries available in various fields. Many have suggested that biases are exhibited more strongly as the prestige of a field, department or position increases. While it is difficult to measure prestige precisely, it often goes hand-inhand with money. Nelson's data show a higher proportion of men in "top" departments ${ }^{11}$ which are usually research-oriented with significant amounts of research funding. Mason and Ekman report that within medicine, women tend to be clustered in the lower paying, lower prestige disciplines ${ }^{4}$. If one looks across engineering fields would one find a similar relationship between the number of women and research funding or salaries?

4. The possibility for true part-time opportunities. Many engineering jobs, whether in academia or industry, do not offer satisfactory part-time opportunities that allow parents time to focus on young families. The engineering profession is under great productivity pressure, and those who opt for part time positions may fear that they will be the first to be cut in the next re-organization.

5. The occurrence of lawsuits within a field or department, such as one that occurred in the Chemistry department at the University of Minnesota, which resulted in a lasting impact on hiring practices in that department for decades afterwards, which continues today.

6. Achievement of a "tipping point" at which a sufficient number of women are present to change the culture and accepted norms of the discipline, and to provide a sufficient pool from which leaders can be chosen. Reaching a tipping point may be an essential prerequisite to change, but judging from the example of psychology where women have long dominated the student population but men still dominate the faculty, numbers alone may not be sufficient to cause an existing status quo to crumble.

7. The existence of female leaders and role models at all levels, including the top. Women leaders serve as role models, and many, but not all, may be champions of changes to improve gender equity.

8. The presence of champions, particularly at the top who tirelessly and systematically champion change. Such champions may be male of female.

\section{Closing Thoughts}

While engineering has changed greatly over the past 40 years, it has still been much slower to change than most other historically male dominated fields. Engineering is still quite far from gender parity, and from an equitable climate that supports both men and women. National data on the percentages of women graduates shows that while the proportion of women has grown in 
most historically male dominated fields, the proportion of women present such fields is closely related to the proportion of women that were present in that field in the 1960's. There were virtually no women in engineering in the 60's and so there are few now. It may take women in a field to attract more women into it. What can engineering do to break away from its past? The author suspects that factors such as increases in number of faculty and leaders (department heads, deans, etc.) at universities and colleges may be key in influencing greater proportions of women to choose engineering as a profession. Targeted investments in this relatively small group may have a large impact on the proportions of women in industry as well, by changing the proportion of women who choose to pursue an engineering education. Additionally, media images and outreach to high schools may be important for helping both young women and men to understand and envision engineering as a career before they select a college or university. Further change will likely require a sustained effort, simultaneously addressing multiple factors. However, change is necessary if engineering is to continue to provide the underpinnings of our economy. Furthermore, if Blum and Frieze' study is any indicator, continued change will likely improve the climate and appeal of engineering for all students.

\section{Bibliography}

1. Figures for 2004, Association of American Medical Colleges (2005) "The Changing Representation of Men and Women in Academic Medicine," Analysis in Brief, 5(2).

2. Figures for 2004 and 2005 respectively, from the National Science Foundation website http://www.nsf.gov/statistics/wmpd/underdeg.htm

3. Valian, V. (1998) Why So Slow? The Advancement of Women. MIT Press.

4. Mason, M. A. and E. M. Ekman (2007) Mothers on the Fast Track: How a New Generation Can Balance Family and Careers, Oxford University Press.

5. Banaji, M. R. and A. G. Greenwald (1995) "Implicit Gender Stereotyping in Judgments of Fame," Journal of Personality and Social Psychology, 68(2):181-198.

6. https://implicit.harvard.edu/implicit/

7. Etzowitz, H., C. Kemelgor, M. Neushatz and B. Uzzi (2000) Athena Unbound: The Advancement of Women in Science and Technology, Cambridge, U. K., Cambridge University Press.

8. Griffin, P. (1997) "Introductory module for the Single Issue Courses," in M. Adams, L.A. Bell, \& P. Griffin, eds. Teaching for Diversity and Social Justice: A Sourcebook, New York: Routledge, pp. 61-109.

9. West, M. A. and J. W. Curtis (2006) "AAUP Faculty Gender Equity Indicators 2006" American Society of University Professors, Washington, D. C. USA.

10. Atkin, A. M.; R. Green and L. McLaughlin, (2002) "Patching the Leaky Pipeline," Journal of College Science Teaching, 32(2):102-108.

11. Nelson, D. (2007) "A National Analysis of Minorities in Science and Engineering Faculties at Research Universities," October 31, 2007. http://cheminfo.ou.edu/ djn/diversity/Faculty_Tables_FY07/FinalReport07.html

12. U.S. Department of Education (2003) "The Condition of Education, 2002," National Center for Education Statistics, Washington D.C., USA.

13. National Science Foundation (2007) "Science and Engineering Degrees: 1966-2004," Division of Science Resources Statistics, January 2007, http://www.nsf.gov/statistics/nsf07307/content.cfm?pub_id=3634\&id=2

14. Blum, L. and C. Frieze (2005) "As the Culture of Computing Evolves Similarity can be the Difference," Gender and IT issue of Frontiers, 26:1 2005.

15. Margolis, J. and Allan Fisher (2003) Unlocking the Clubhouse: Women in Computing, MIT Press, Cambridge MASS, USA.

16. Nelson, D. (2004) “A National Analysis of Diversity in Science and Engineering Faculties at Research Universities." http://cheminfo.ou.edu/ djn/diversity/Faculty_Tables_FY07/FinalReport07.html

17. Marschke, R; S. Laursen; J. Neilson and P. Rankin (2007) "Demographic Inertia Revisited: An Immodest Proposal to Achieve Equitable Gender Representation among Faculty in Higher Education," the Journal of Higher Education, 78(1):1-26. 